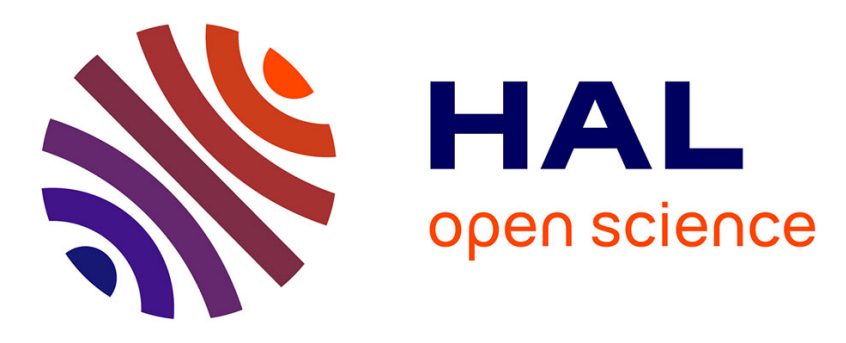

\title{
Credibilist Occupancy Grids for Vehicle Perception in Dynamic Environments
}

Julien Moras, Véronique Cherfaoui, Philippe Bonnifait

\section{To cite this version:}

Julien Moras, Véronique Cherfaoui, Philippe Bonnifait. Credibilist Occupancy Grids for Vehicle Perception in Dynamic Environments. IEEE International Conference on Robotics and Automation (ICRA 2011), May 2011, Shanghai, China. pp.84-89. hal-00615303

\section{HAL Id: hal-00615303 https://hal.science/hal-00615303}

Submitted on 18 Aug 2011

HAL is a multi-disciplinary open access archive for the deposit and dissemination of scientific research documents, whether they are published or not. The documents may come from teaching and research institutions in France or abroad, or from public or private research centers.
L'archive ouverte pluridisciplinaire HAL, est destinée au dépôt et à la diffusion de documents scientifiques de niveau recherche, publiés ou non, émanant des établissements d'enseignement et de recherche français ou étrangers, des laboratoires publics ou privés. 


\title{
Credibilist Occupancy Grids for Vehicle Perception in Dynamic Environments
}

\author{
Julien Moras, Véronique Cherfaoui, Philippe Bonnifait
}

\begin{abstract}
In urban environments, moving obstacles detection and free space determination are key issues for driving assistance systems and autonomous vehicles. When using lidar sensors scanning in front of the vehicle, uncertainty arises from ignorance and errors. Ignorance is due to the perception of new areas and errors come from imprecise pose estimation and noisy measurements. Complexity is also increased when the lidar provides multi-echo and multi-layer information. This paper presents an occupancy grid framework that has been designed to manage these different sources of uncertainty. A way to address this problem is to use grids projected onto the road surface in global and local frames. The global one generates the mapping and the local one is used to deal with moving objects. A credibilist approach is used to model the sensor information and to do a global fusion with the worldfixed map. Outdoor experimental results carried out with a precise positioning system show that such a perception strategy increases significantly the performance compared to a standard approach.
\end{abstract}

\section{INTRODUCTION}

There is a strong interest in the field of intelligent systems and robotics to develop autonomous vehicles with the ability to navigate in challenging environments. Indeed, several large-scale experiments like the DARPA Urban Challenge [1] or the new challenge VIAC [2] (4 vehicles from Italy to China) have shown impressive results. However, autonomous driving in urban areas remains a problem and needs scene understanding capabilities to predict the short-term evolution for reliable navigation. For road navigation applications, safety is one of the fundamental requirements. A mere detection of the obstacles is not enough: a fine characterization of the navigable space is necessary to plan a safe motion of the robot [3]. For a car-like robot, navigable space means all the drivable space in the surrounding of the vehicle and obstacle means a insurmountable part of the space. This information has to be provided in real time with high level of confidence.

Some sensors providing high density information, for example the Velodyne [4], are able to attempt the necessary level of confidence for navigation. But it requires perception systems that are capable of handling its high bandwidth or processing requirements. However, an other solution consists in using sensor providing sparse data. Representation of the environment is obtained exploiting temporal redundancy and temporal complementary of data.

In robotics, mapping (M) is coupled generally with localization in the Simultaneous Localization And Mapping (SLAM) problem [5], [6]. Two main approaches exist: the

The authors are with Heudiasyc,CNRS UMR 6599 , Université de Technologie de Compiègne, 60200 Compiègne, France Contact author email: julien.moras@hds.utc.fr feature-based approach which tries to map with a predetermined set of shapes (segment, arc, etc...) [7] and the grid approach which is based on a discrete space representation [8]. SLAM methods are sensitive to the presence of moving objects in the scene. Indeed, algorithms are based on the temporal consistency of the map. A way to address this issue is to perform detection and Mobile Objects Tracking (MOT) [9]. Usually, a detection and tracking mechanism is used in the feature-based framework. It works in 3 steps: clustering of raw data for object detection, data association and temporal fusion [10]. The main sources of error are in the clustering phase and in the association step [11]. Some recent works use parametric models to follow the variation of the shape of the objects [12], [13].

This paper presents an approach dedicated to mapping and detection of moving obstacles using a sensor observing just a part of the environment. This kind of range sensor provides two types of information: the presence of echoes in the lineof-sight, and their relative position. The main contributions of this paper are the definition of a new sensor model adapted to a grid representation and a method to extract moving objects while performing the mapping using a credibilist fusion. As a clustering step is no longer necessary, there is no assumption on the shape of the objects, making it suitable for a wide range of urban objects (pedestrians, vehicles, bicycles ,...). Moreover, partially observed objects because of occlusions or sensor field of view limitation can be more precisely localized. The accurate pose of the robot is not a study of this paper and is provided by a specific module.

The paper is organized as follows. In section II, we present the occupancy grid. We then introduce the credibilist grids proposed in this work. Afterward, the sensor model is presented in section III, followed by the fusion processing stage in section IV. Finally, experimental results are given and analyzed in section V.

\section{CRedibilist OCCUPANCY GRIDS}

\section{A. Grid representation}

An occupancy grid is a representation of the environment which proposes to split the space into a set of cells that may be free or occupied. Usual approaches attempt to determine the probability that every cell is occupied from sensor data. The grid framework was proposed by Elfes [14], but it remained little used at that time because this approach is time, memory and computing consuming. During the recent years, this representation has become more and more used because it is an efficient framework for vehicle navigation since it represents in an explicit way the navigable 
space. Moreover, embedded computers have more powerful calculation capabilities (memory, multi-core, GPU, etc...).

Bayesian inference is the common background used to cope with errors and uncertainty. Many extensions have been published in the literature, like the Bayesian Occupancy Filter (BOF) [15] which estimates simultaneously the occupation and the speed of the cells. Some works add prior information to the BOF in order to model mobile object motions [16]. The grid framework has currently been used to solve the complete perception problem from a mobile platform point of view in a dynamic environment [17], since it is able to represent explicitly the free space and the locations of the obstacles.

In the present work, two grids are managed simultaneously: a ScanGrid (SG) which captures the current sensor information and a MapGrid (MG) performing the temporal integration of data in a fixed frame.

\section{B. A credibilist framework to combine sensor data}

In this work, we propose to use a Credibilist approach based on Dempster-Shafer (DS) theory because this framework offers an interesting solution to make the difference between unknown (there is no information) and doubt caused by conflicting information gathered incrementally in the fusion process. Similar ideas have been developed in [18] to implement a DS (also called Evidential) occupancy grid intended to manage in the representation of the environment the specific uncertainty arising from the ultrasonic sensor used. Unfortunately, the DS fusion operator used in that work was very time consuming and showed a important sensitivity to aberrant measurements.

Let consider a concrete case to illustrate these concepts. There is an undiscovered cell in the map and a cell that has been observed free previously and that is now occupied by a moving object. When using a Bayesian framework, these two cells will have the same belief after the measurement update, e.g. an occupancy probability equal to 0.5 . In a credibilist framework undiscovered cells receive a belief mass on a special state called "unknown" whereas in the other cases (appearance or disappearance of an object) lead to produce a belief mass on what is called "conflict".

Let see how these concepts are handled. In a way similar to probabilistic occupancy grids, every grid cell is assigned a state between two possible values Free $(F)$ and Occupied $(O)$. Let defined $\Omega$ the ignorance state in the same way that in [18], e.g. $\Omega=\{F, O\}$. In the theory of belief functions, one has to increase this set, by considering the power-set which is defined as $2^{\Omega}=\{F, O, \Omega, \emptyset\}$. For each cell, a mass function is calculated and provides four beliefs on the state of the cell $[m(F) m(O) m(\Omega) m(\emptyset)]$, where $m(A)$ represents respectively the piece of evidence that the space is free, occupied, unknown or resulting of conflict. Mass functions verify the property $\sum_{A \subset \Omega} m(A)=1$.

The Transferable Belief Model (TBM) framework proposed by Smets [19] provides also powerful tools since many fusion operators were developed in order to cope with different problems. The conjunctive combination rule, noted
(D), is the one that is the most well known and treats the case of the fusion of two reliable sources. It is defined as follows. Let $m_{1}$ and $m_{2}$ be two given mass functions and let $m_{1} \bigcirc 2$ be the result of their combination by (). The result of the combination using conjunctive rule is computed in the following way:

$$
\forall A \subseteq \Omega, m_{1} \bigcirc 2(A)=\sum_{B \cap C=A \mid B, C \subseteq \Omega} m_{1}(B) \cdot m_{2}(C)
$$

In the case of undiscovered cells by both sources $S_{1}$ and $S_{2}$, (Eq. 1) gives the Basic Belief Assignments (BBA). The result of the combination (Eq. 2) shows that the mass remains on $\Omega$.

$$
\begin{aligned}
& \forall i \in[12] \quad m_{i}(F)=0 \quad m_{i}(O)=0 \\
& m_{i}(\Omega)=1 \quad m_{i}(\emptyset)=0 \\
& m_{1} \bigcirc_{2}(F)=0 \quad m_{1} \bigcirc_{2}(O)=0 \\
& m_{1} @ 2(\Omega)=1 \quad m_{1} @ 2(\emptyset)=0
\end{aligned}
$$

In the case of two opposite observations of the sources $S_{1}$ and $S_{2}$, the combination of the two BBA (Eq. 3) shows that the resulting mass is concentrated on the empty set illustrating the conflicting situation (Eq. 4).

$$
\begin{array}{cccc}
m_{1}(F)=0 & m_{1}(O)=0.8 & m_{1}(\Omega)=0.2 & m_{1}(\emptyset)=0 \\
m_{2}(F)=0.8 \quad m_{2}(O)=0 & m_{2}(\Omega)=0.2 & m_{2}(\emptyset)=0 \\
& \\
m_{1} @_{2}(F)=0.16 & m_{1} @_{2}(O)=0.16 \\
m_{1} \bigcirc_{2}(\Omega)=0.04 & m_{1} \bigcap_{2}(\emptyset)=0.64
\end{array}
$$

Contrary to a probabilistic approach, the difference between the two cases is significant. Several works have emphasized the importance of conflict analysis [20], [21]. This particular point is a key issue in our approach.

\section{FROM SENSOR DATA TO CREDIBILIST SCANGRID}

In this section, we explain how to build a credibilist SG in polar coordinates using a multi-layer lidar scan with potentially several echoes in the same line of sight.

\section{A. Sensor model}

Sensors provide data from physical phenomena regardless of the contextual situation. To interpret such information, one has to model the sensor by making some simplifying assumptions. In this paper, a lidar sensor is used and we assume that the different laser beams are above the road i.e. the echoes are coming from potential obstacles. In order to be as close as possible to the sensor acquisition process, the $\mathrm{SG}$ is created in a polar frame.

The space is divided in several angular sectors as shown in Figure 1. Multiple measures in the same angular sector are possible because the sensor provides multi-echo measurement in several layers.

On one hand, each sector is considered independently of the others. This allows computing the SG row by row (1 row $=1$ sector). On the other hand, each cell in a row depends on the other ones. This is due to the propagation of the laser beam and also to the multi-echoes strategy, since the state of cells in a row depends on their position compared to the echo position. Figure 2 illustrates the row affectation according to several lidar measurements in a sector. 


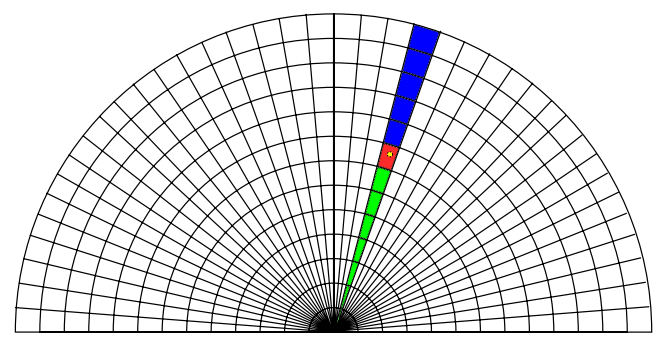

Fig. 1. Polar space representation, showing how the information from a measurement can be interpreted. Green refers to free space, red to occupied and blue to unknown .

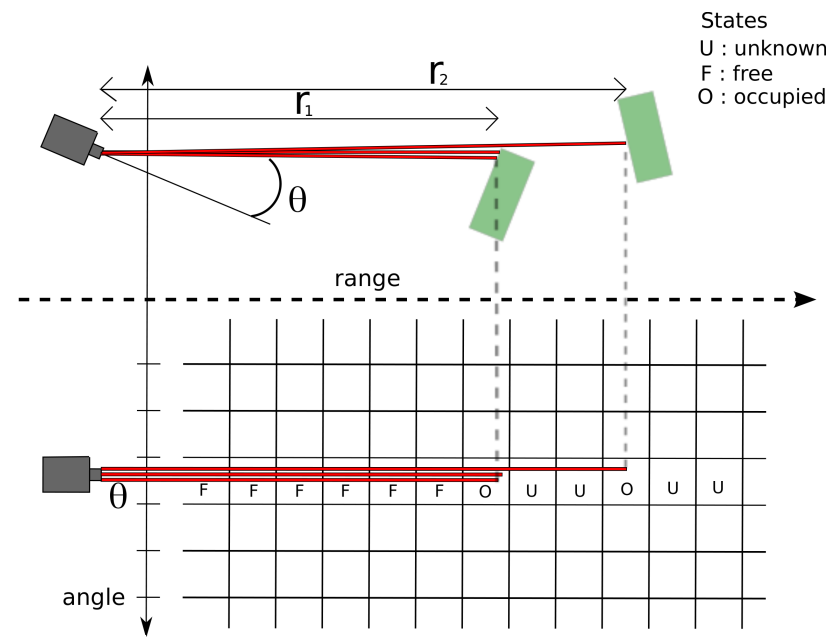

Fig. 2. ScanGrid Construction, top figure is a bird's eye view of the sensor. Bottom figure plots the conceptual SG row state assignment.

\section{B. ScanGrid computation}

This section details the construction of one row of the $\mathrm{SG}$, corresponding to the angular sector $\Theta=\left[\theta^{-}, \theta^{+}\right]$. We define a BBA for each cell computed from the sensor scan points. One cell is a box $R \times \Theta, R=\left[r^{-}, r^{+}\right]$. Let $Z_{\Theta}=\left\{z_{i}=\left[\begin{array}{c}r_{i} \\ \theta_{i}\end{array}\right], \theta_{i} \in \Theta, i \in[0, n]\right\}$ the set of $n$ scan points in the angular sector $\Theta$. BBAs $m_{S}$ are performed in the following way.

for every cell $\{R, \Theta\}$ :

$-\forall i \in[1, n]$ if $\exists r_{i} \mid r_{i} \in R$ then

$$
m_{S}(\emptyset)=0 \quad m_{S}(F)=0 \quad m_{S}(O)=1-\lambda_{O} \quad m_{S}(\Omega)=\lambda_{O}
$$

$-\forall i \in[1, n]$ if $\nexists r_{i} \mid r_{i} \in R$ then

$$
\begin{gathered}
m_{S}(\emptyset)=0 \quad m_{S}(F)=1-\lambda_{F} \quad m_{S}(O)=0 \quad m_{S}(\Omega)=\lambda_{F} \\
-\forall i \in[1, n] \text { if } \nexists r_{i} \mid r_{i} \in R \text { and } r^{+}<\min \left(r_{i}\right) \text { then } \\
m_{S}(\emptyset)=0 \quad m_{S}(F)=0 \quad m_{S}(O)=0 \quad m_{S}(\Omega)=1
\end{gathered}
$$

$\lambda_{F}$ and $\lambda_{O}$ are parameters belong to $[0,1]$ and reflect the confidence in the measurement ( 0 if confident). This confidence is linked to the principle of measurement (false alarm or miss detection). Indeed, the angular separation between two points is larger than the width of a laser beam. So, there is a nonzero probability that an object is not detected as explained in Figure 3.

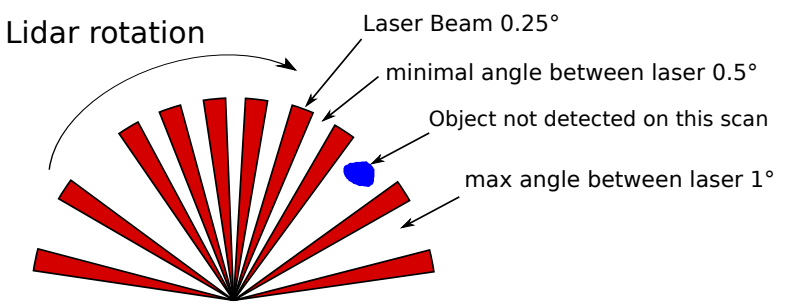

Fig. 3. Case of no detected object with lidar

It is important to notice that the non scanned angular sectors are fully affected with the vacuous mass $m_{\Omega}$ (Eq. 5 ) in order to indicate that there is no information.

$$
m_{\Omega}(F)=0 \quad m_{\Omega}(O)=0 \quad m_{\Omega}(\Omega)=1 \quad m_{\Omega}(\emptyset)=0
$$

\section{GLOBAL FUSION AT THE MAPPING LEVEL}

Grid transformations, map updating and mobile objects detection are described in this section.

\section{A. System overview}

Our aim is to build a grid in a global world frame (MapGrid) to map the navigable space and the static environment. The MG is a Cartesian map referenced in a world frame and composed of $L$-length squared cells. Each cell refers to an occupancy mass function defines on $2^{\Omega}$ as described in section II-B. $m_{M_{i, j}, t}$ is the mass function referring to the cell $(i, j)$ of MG at time $t$.

The MapGrid is initialized using no prior information, e.g. with vacuous mass $m_{\Omega}$ on every cell.

The updating mechanism is sequential. At a given time, the previous MapGrid, MG(t-1), is updated with the current ScanGrid $\mathrm{SG}(\mathrm{t})$ built from the sensor measurements. The result is a new MapGrid MG(t). The updating step allows classifying cells containing moving objects. The proposed scheme is composed of four phases as described by Figure 4.

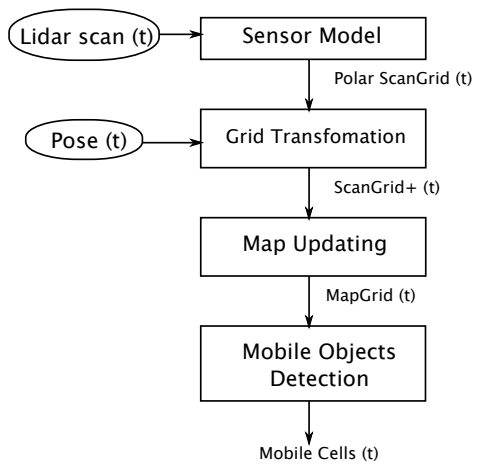

Fig. 4. Grid based perception scheme for mapping and moving objects detection

\section{B. Grid transformation}

To be able to merge data from the SG, one has to transform the grid support to be spatially compatible with the MapGrid. This is achieved in two stages. First, the SG is converted from polar to Cartesian coordinates, then it is 
moved from the sensor frame into the reference frame of MapGrid. This requires the absolute pose of the vehicle at time $t$ to be available. Both transformations are achieved by using bi-linear interpolation methods on each of piece of evidence of mass functions. In practice, the grid can be regarded as a multi-channel image, which allows the use of image processing tools. The result is a credibilist grid called ScanGrid+ (SG+) and mass function is noted $m_{S^{+}, t}$.

\section{Global map update with the ScanGrid}

When a new ScanGrid+ $\mathrm{SG}+(\mathrm{t})$ is computed, we fuse it with the previous MapGrid MG(t-1). Every cell is considered independent from the others.

The fusion is performed using the Dempster combination rule, but it is achieved in two steps in order to keep the conflicting information.

We fuse with a conjunctive rule (Eq. 6) and we store the empty set mass $m^{\prime}(\emptyset)$ for a further process. As the fusion is done element by element across the entire grid, we do not repeat the indices of the cells: $m_{M, t}$ refers to $\forall i, j, m_{M_{i, j}, t}$. Then the empty-set mass is used for normalization (Eq. 7).

$$
\begin{gathered}
m_{M, t}^{\prime}=m_{M, t-1} @ m_{S^{+}, t} \\
\begin{cases}m_{M, t}(A)=\frac{m_{M, t}^{\prime}(A)}{1-m_{M, t}^{\prime}(\emptyset)} & A \neq \emptyset \\
m_{M, t}(\emptyset)=0 & A=\emptyset\end{cases}
\end{gathered}
$$

Figure 5 shows the behavior of the mass function of one cell belongs to the MG along the process composed of six situations.

In the first step, the MG cell, initialized with the vacuous mass, converges to a free state according to the SG+. Then during step 2, since no change occurs, it remains stable. Step 3 is the beginning of a change in the SG+ from $F$ to $O$. Two contradictory pieces of information create a part of conflict, the other part of the mass remains on $F$. When $\mathrm{SG}+$ remains on the same state, it forces the MG to change from $F$ to $O$ as shown by step 4 . The following steps 5 and 6 do the inverse process.

\section{Mobile object detection}

If MG and SG+ contradicts themselves conflict occurs. A mobile object detection is done by analyzing the conflicting mass. This mass is computed by:

$m_{M, t}^{\prime}(\emptyset)=m_{M, t-1}(O) \cdot m_{S^{+}, t}(F)+m_{M, t-1}(F) \cdot m_{S^{+}, t}(O)$

It is composed of two different parts.

- The term $m_{M, t-1}(F) \cdot m_{S^{+}, t}(O)$ represents the conflict produced by the fusion of an occupied cell of the SG+ with a free cell of the MG. This can occur when a moving object appears in the cell.

- The term $m_{M, t-1}(O) \cdot m_{S^{+}, t}(F)$ represents the conflict produced by the fusion of a free cell of the SG+ with an occupied cell of the MG. This can occur when a moving object leaves the cell.

It may be noted that the conflict is generated for a short time, because if an object remains in a new cell, it will gradually change the MG and will be considered as static.

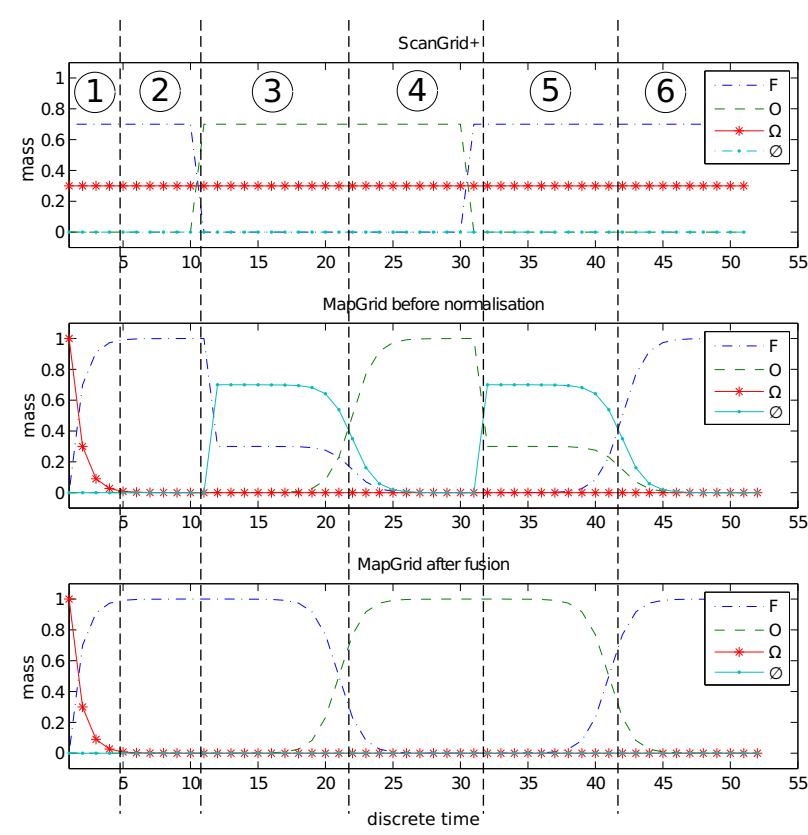

Fig. 5. Simulation of the evolution of the mass functions. Top figure: simulated ScanGrid+ detection. Center figure: result of the conjunctive combination. Bottom figure: result of Dempster's rule.

\section{EXPERIMENTAL RESULTS}

The presented algorithm was tested on a data-set acquired with an equipped vehicle in urban conditions and was compared to another approach based on accumulation operators [22]. The data-set is a 20-minutes long sequence, acquired in collaboration with the French Geographic Institute (IGN) in Paris. The vehicle (left Fig. 6) follows a reference track (Right Fig. 6) repeated 3 times. The results presented were conducted during the first loop.

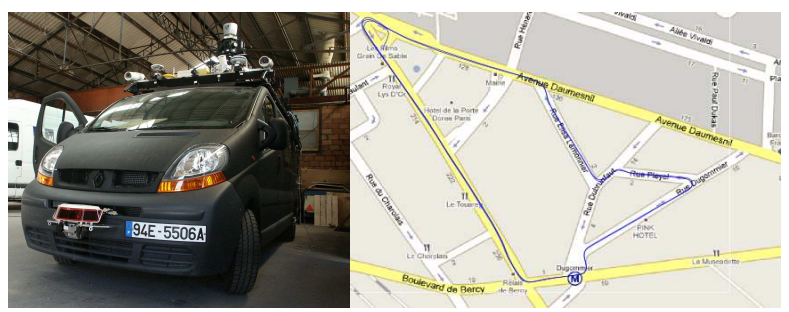

Fig. 6. Left: the test vehicle with the lidar sensor in front. Right: the track followed by the vehicle

Two sensors were used: Lidar ALASCA XT and Applanix positioning system. To synchronize the data, time stamps in GPS time were used. The lidar was installed to have its lowest layer horizontally: if we assume a low pitch, the floor was not detected by lidar. The lidar frequency was $15 \mathrm{~Hz}$ with a maximum angular resolution of $0.25^{\circ}$ in the vehicle front and $1^{\circ}$ on the sides. The Applanix positioning data was post-processed in order to provide a precise pose. A camera was installed just below the lidar located on the front bumper. Images were not processed, they were just used for validation and visualization of the scene. 
The SG reaches a distance of $100 \mathrm{~m}$ with a $180^{\circ}$ angular aperture and a resolution of $0.5 \mathrm{~m} \mathrm{x} 1^{\circ}$ angle. MG covers an area of $800 \mathrm{~m} \times 700 \mathrm{~m}$ with a resolution of $0.5 \mathrm{~m} \times 0.5 \mathrm{~m}$.

An offline implementation of the algorithm has been developed with Matlab and a real time version ( $\mathrm{C}++)$ is under development.

The coefficients $\lambda_{F}$ and $\lambda_{o}$ have been set at 0.5 and the detection threshold on the conflict for mobile objects detection at 0.1 .

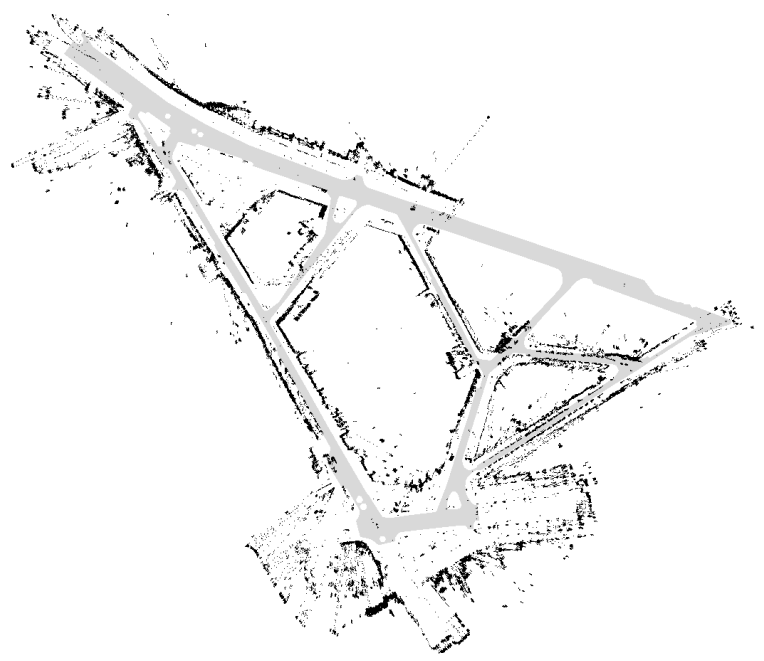

Fig. 7. Result of an accumulation mapping, black refers to occupied cells, gray refers to the road space provided by IGN

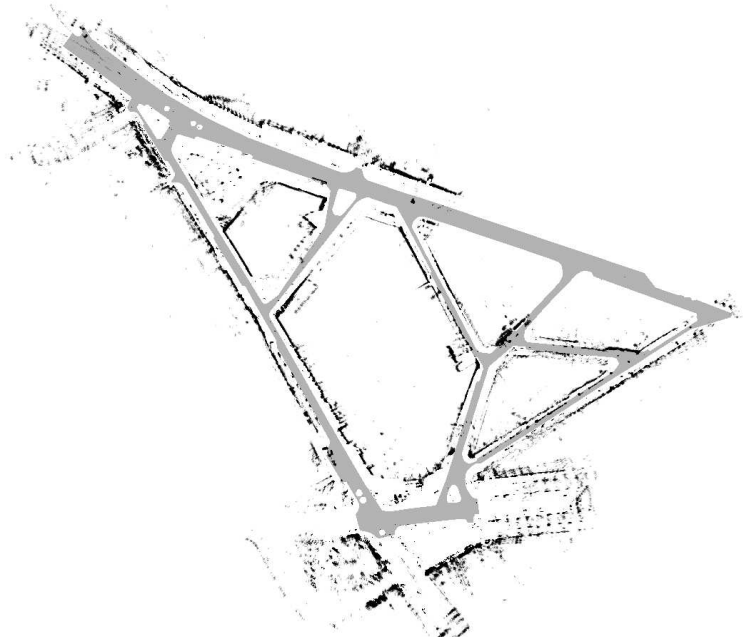

Fig. 8. Result of the credibilist mapping, black refers to occupied cells, gray refers to the road space provided by IGN

Static environment mapping: Figure 7 draws the mapping results of a previously implemented method based on accumulation [22]. Figure 8 draws the mapping results of the credibilist method in the same conditions. The mapping was performed in real traffic condition, i.e. with the presence of many cars and pedestrians. In order to evaluate the quality of the mapping, we use a map of road space provided by IGN (Fig. 7,8: gray cells). The black cells outside the gray area represent the building. They are mapped in the same way with both methods. The accumulation approach presents many occupied cells scattered on the free space. This phenomenas are due to moving objects. The credibilist framework is less affected because this approach offers a better management of the moving objects. Indeed, the conflict analysis allows the detection of moving objects without mapping them. So, the updating mechanism performs a filter rejecting moving objects and outliers measurements while taking advantage of redundancy. The accumulation scheme adds mobile objects into the map and clean them when they leave the place. If one object is not observed when it leaves a cell, this cell remains occupied.

Moving object detection: Figure 9 illustrates results of both method to detect mobile objects during a small sequence. This sequence shows two cars passing in front of the ego-vehicle from the right then a truck crossing from the left. As there is no clustering, the vehicle was manually circled with the same color on the camera frame and on the maps in order to identify them. Maps are displayed in global frame (MG) and give a bird view of the scene. The ego-vehicle is represented by the yellow triangle looking at the top left side.

First, let focus on the accumulation approach (middle plots on each step). On this sequence, it presents poor results. Indeed, for each vehicle, even if the front is partially detected as mobile (green cells), they are mostly miss classified (blue cells refer to static objects). We can also see that mobile objects were propagated into the map during such a time (for example: black cells on the truck). The results may be made better by tuning the classification threshold, but this will create false alarms on real static elements classified as mobile.

The credibilist approach (low plot on each step) shows correct classification on each vehicle. Mobile objects don't remain enough time on the cells to change the map. The main drawback of the method is the false alarms on static objects due to the map transformation and on free space due to outliers measurement. We can notice that with both methods, objects moving in masked area are directly mapped and not classified (cluster of occupied cells in top right for example). Finally, one can conclude that the analysis of the conflict improves greatly the performance.

\section{CONCLUSION}

This paper has presented a perception fusion scheme based on both local and global grid interactions. The main contribution of this work has been to develop and test a credibilist fusion approach which provides the navigation space, the mobile obstacles and the static objects. Experimental results reported here illustrate the good performance of such a strategy for detecting mobile objects which is a crucial stage in perception. A credibilist framework seems to be an interesting way to achieve such kind of simultaneous mapping and moving objects localization using grids. Indeed, a particular attention can be given to the distinction of total ignorance with conflict arising from movements in the scene. 

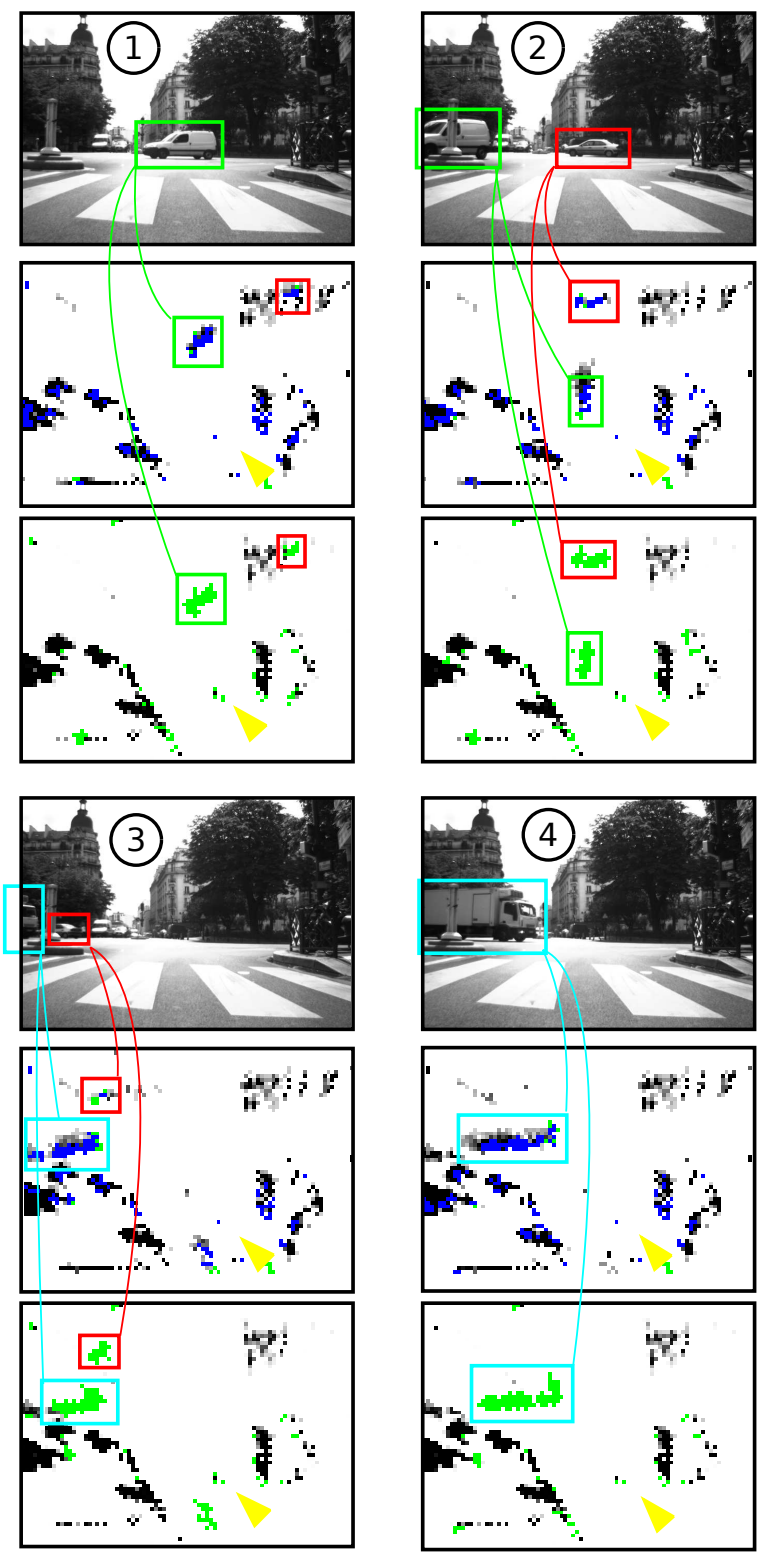

Fig. 9. Result of the detection of moving objects, four snapshots of the sequence. Each snapshot is illustrated by a camera image of the scene and the local map displaying the moving objects. For the accumulation approach (middle) the blue refers to static and the green refers to moving. In the credibilist approach (bottom), green refers to $m(\emptyset)$, the moving objects.

We have noticed a deep performance improvement compared to accumulation strategies that are quite difficult to tune and which often fail to detect large moving objects. Moreover, this strategy has shown to be very efficient for capturing pieces of information coming from all the lidar's echoes by explicitly giving no occupancy information between the echoes.

In its current implementation, precise localization is a prerequisite. One perspective is to analyze how our approach is robust to a dead-reckoning localization method, using for instance lidar odometry. Another perspective is to use another credibilist sensor model able to fuse each sensor measurement independently.

\section{ACKNOWLEDGMENTS}

This work is supported by the French ANR CityVip project. The system has been tested on sequences recorded by the test platform "STEREOPOLIS".

\section{REFERENCES}

[1] M. Buehler, K. Iagnemma, and S. Singh, Eds., The DARPA Urban Challenge: Autonomous Vehicles in City Traffic, George Air Force Base, Victorville, California, USA, ser. Springer Tracts in Advanced Robotics, vol. 56. Springer, 2009.

[2] A. Broggi, L. Bombini, S. Cattani, P. Cerri, and R. Fedriga, "Sensing requirements for a $13,000 \mathrm{~km}$ intercontinental autonomous drive," Intelligent Vehicles Symposium (IV), 2010 IEEE, 2010.

[3] M. Himmelsbach, T. Luettel, F. Hecker, F. von Hundelshausen, and H.-J. Wuensche, "Autonomous off-road navigation for mucar-3," KI KÃijnstliche Intelligenz, pp. 1-5, 2011.

[4] M. Himmelsbach, F. v. Hundelshausen, and H. Wuensche, "Fast segmentation of $3 \mathrm{~d}$ point clouds for ground vehicles," in Proc. IEEE Intelligent Vehicles Symp. (IV), 2010, pp. 560-565.

[5] Y. Zhao, H. Chiba, M. Shibasaki, R. Shao, X. Cui, and J.Zha, "Slam in a dynamic large outdoor environment using a laser scanner," in IEEE Int. Conf. on Robotics and Automation (ICRA), 2008.

[6] S. Thrun, W. Burgard, and D. Fox, Probabilistic Robotics (Intelligent Robotics and Autonomous Agents), 2001.

[7] W. Y. Jeong and K. M. Lee, "Visual slam with line and corner features," in Proc. IEEE/RSJ Int Intelligent Robots and Systems Conf, 2006, pp. 2570-2575.

[8] O. Garcia-Favrot and M. Parent, "Laser scanner based slam in real road and traffic environment," in IEEE International Conference Robotics and Automation (ICRA09). Workshop on Safe navigation in open and dynamic environments Application to autonomous vehicles, 2009.

[9] C. C. Wang, C. Thorpe, S. Thrun, M. Hebert, and H. Durrant-Whyte, "Simultaneous localization, mapping and moving object tracking," The International Journal of Robotics Research, vol. 26, no. 9, pp. 889916, September 2007.

[10] Y. Bar-Shalom, Multitarget-Multisensor tracking : Applications and Andvances. Artech House, 2000.

[11] S. Blackman and R. Popoli, Design and Analysis of Modern Tracking Systems. Artech House, 1999.

[12] A. Petrovskaya and S. Thrun, "Model based vehicle detection and tracking for autonomous urban driving," Auton. Robots, vol. 26, no. 2-3, pp. 123-139, 2009.

[13] F. Fayad and V. Cherfaoui, "Tracking objects using a laser scanner in driving situation based on modeling target shape," IEEE Intelligent Vehicles Symposium, 2007.

[14] A. Elfes, "Using occupancy grids for mobile robot perception and navigation," Computer, vol. 22, no. 6, pp. 46 - 57, 1989.

[15] C. Coue, C. Pradalier, C. Laugier, T. Fraichard, and P. Bessiere, "Bayesian occupancy filteing for multitarget tracking : an automotive application," International Journal of robotics research, vol. 25, no. 1, pp. 19-30, 2006

[16] T. Gindele, S. Brechtel, J. Schröder, and R. Dillmann, "Bayesian occupancy grid filter for dynamic environments using prior map knowledge," Intelligent Vehicles Symposium, 2009 IEEE, 2009.

[17] G. Gate, "Reliable perception of highly changing environments Implementations for car-to-pedestrian collision avoidance systems," Ph.D. dissertation, Ecole Nationale Superieure Des Mines De Paris, 2009.

[18] D. Pagac, E. M. Nebot, and H.Durrant-Whyte, "An evidential approach to map-building for autonomous vehicles," IEEE Transactions on Robotics and Automation, vol. 14, no. 4, pp. 623-629, 1998.

[19] P. Smets and R. Kennes, "The transferable belief model," Artificial Intelligence, vol. 66, pp. 191-234, 1994.

[20] P. Smets, "Analyzing the combination of conflicting belief functions," Inf. Fusion, vol. 8, no. 4, pp. 387-412, 2007.

[21] E. Ramasso, M. Rombaut, and D. Pellerin, "State filtering and change detection using tbm conflict. application to human action recognition in athletics video." IEEE transaction on circuits and systems for video technology (CSVT), vol. 17, no. 7, pp. 944-949, 2007.

[22] J. Moras, V. Cherfaoui, and P. Bonnifait, "A lidar Perception Scheme for Intelligent Vehicle Navigation," in 11th International Conference on Control, Automation, Robotics and Vision, Singapore, 122010. 